\title{
Digital Chunk Processing with Orthogonal GFDM Doubles Wireless Channel Capacity
}

\author{
Mohammad R. Kadhum, Triantafyllos Kanakis, Ali Al-Sherbaz and Robin Crockett \\ Faculty of Arts, Science \& Technology, University of Northampton, U.K. \\ Email: \{mohammad.kadhum, triantafyllos.kanakis, ali.al-sherbaz, robin.crockett \}@ northampton.ac.uk
}

\begin{abstract}
A novel physical layer (PHY) transmission technique for increasing the channel capacity of transmission, termed as Orthogonal Generalized Frequency Division Multiplexing (OGFDM), has been proposed, investigated and evaluated in this paper. A combination of the Digital Hilbert Filter (DHF) with Generalized Frequency Division Multiplexing (GFDM) has been shown to double wireless channel capacity for each transmitted frequency sub-carrier at acceptable Bit Error Rate (BER) limits. By making use of the great properties of Hilbert transforms, orthogonality is achieved between the traditionally non-orthogonal GDFM subcarriers improving the BER and wireless channel capacity of the transmission. The OGFDM seems to combine the attributes of GFDM and Orthogonal Frequency Division Multiplexing (OFDM) in one sustainable system. The proposed solution achieves orthogonality between the filters of adjacent frequencies of subcarriers instead of between the frequencies of subcarriers themselves. Also, an OGFDM system model is presented, based on which, the relation between the main filter parameters and the system BER and channel capacity performance is specified in a wireless electrical back-to-back transmission system. Finally, by means of simulations, the impact of applying the proposed advanced filters on the aggregated system performance of the BER and channel capacity is shown in an Additive White Gaussian Noise (AWGN) wireless channel.
\end{abstract}

Keywords-Generalized Frequency Division Multiplexing (GFDM); Digital Hilbert Filter (DHF); Orthogonal Frequency Division Multiplexing (OFDM); Wireless channel; Orthogonal Generalized Frequency Division Multiplexing (OGFDM)

\section{INTRODUCTION}

Research of wireless communications has been concentrated in increasing the transmission channel capacity since the $2 \mathrm{G}$ era onwards. Nevertheless, the "data hungry" applications of the modern lifestyle are driving the market making the minimum operational requirements for the future communication networks in need for even higher channel capacities [1]. The main foreseen scenarios for expected networks are Bit pipe Communication (BC) [1], Wireless Regional Area Network (WRAN) [2], Tactile Internet (TI) [3] and Machine Type Communication (MTC) [4]. Orthogonal Frequency Division Multiplexing (OFDM) is the most commonly used channel modulation as a result of its special capabilities with multipath channels and easy implementation using Fast Fourier Transform (FFT) [5]. The recent requirements for elastic networks pose a big challenge for the OFDM structure which suffers from various limitations in addressing the modern needs. For example, high Spectral Efficiency (SE) for the $\mathrm{BC}$, low latency for both the TI and WRAN and low power consumption for the MTC. All those issues due to high Out Of Band (OOB) emissions, resources wasted for Cyclic Prefix
(CP) and orthogonality of the OFDM subcarriers. For those reasons, "OFDM is not the most promising waveform for the next generation networks" [6]. To address this challenge, research has recently started investigating alternative multicarrier systems to be used with the upcoming wireless communication systems. One of the most explored multi-carrier systems is Filter Bank Multi-Carrier (FBMC), where, each frequency sub-carrier is individually filtered to reduce the OOB emissions. Nevertheless, narrow Bandwidth (BW) of each filtered frequency sub-carrier increases the impulse response length of transmission filters resulting in a poor SE unless long bursts are used for transmission. This obviously cannot be considered as a reasonable solution for the innovative network design. In particular, low latency requires a high SE with a small number of transmitted samples [7]. Another proposed candidate waveform is Universal Filtered MultiCarrier (UFMC), in which, the OOB emissions is reduced by filtering a set of transmitted frequencies of subcarriers. Because the BW of the filter can cover a number of transmitted frequencies of subcarriers, a short impulse response can be achieved, which means, reaching good SE with a short burst of transmissions. The main disadvantage of this waveform is its sensitivity against small misalignment of time due to the filtering operation. Therefore, the UFMC is not suitable for the epochal applications, especially, which tend to destroy the time synchronization to keep the energy [8]. The third candidate waveform is Filtered OFDM (F-OFDM), which is very similar to the UFMC but with more flexibility in dealing with multi-user applications. However, the key disadvantages of the UFMC still accompany to the F-OFDM [9]. Recently, Generalized Frequency Division Multiplexing (GFDM) has been proposed as a new candidate waveform for the upcoming wireless and cellular communication networks. Its capability in addressing most of the requirements of the modern telecommunication networks presents it as a promising solution for their Physical layer (PHY) [10], [11]. The small overhead which results from attaching a single CP for each GFDM block improves the SE and the synchronization requirements for the MTC application. Besides, to reduce the OOB emissions, the frequency sub-carrier of the GFDM is filtered circularly in time and frequency domain. Moreover, the GFDM is compatible with the short latency requirements of the demanding realtime applications by reducing the impulse length of its filters to be shorter than the other proposed candidates. Thus, it is considered as an efficient base for the most candidate waveform for the future generation of Mobile. However, the filtering operation of the GFDM affects the criteria of orthogonality for the transmitted frequencies of subcarriers and increases both 
Inter-Carrier Interference (ICI) and Inter-Symbol Interference (ISI) [10]. For these reasons, the proposed candidate waveform in this paper focuses on improving the filtering process by employing a new kind of advanced digital filters able to address the orthogonality and the OOB issues efficiently. Despite the increased computational complexity of the applied filters, todays technology is already able to manage it sufficiently in particular with the multi-core systems. A flexible, powerful and customisable simulation has been developed to supply a good experimentally proof of the proposed concept. Besides, the main system parameters are presented to indicate key configuration for the proposed transceiver. The remaining sections are constructed as follows: Section II explores theoretically the main principles of the newly developed system, highlighting the major physics and maths beyond it. Section III explain experimentally how the proposed solution can address the current insufficient performance of the GFDM in terms of the channel capacity and level of errors. Eventually, section IV concludes the paper.

\section{OGFDM SYSTEM MODEL}

In this paper, a developed GFDM design is introduced. It is capable to improve channel capacity and Bit Error Rate (BER) performance of the system yet keeping the same level of computational complexity of the conventional GFDM. This is achieved by demonstrating a filtering operation using a durable kind of filter able to address the drawbacks of the original GFDM filter. As shown in Fig. 1, a digital input sequence is converted to the frequency domain using Phase Shift Keying (BPSK) modulation scheme. The adjustable signal is consequently up-sampled by a factor of $K$, denoted by ( $K \uparrow$ ), padding $K-1$ null samples (zeros) between adjacent samples. Thereafter, the up-sampled frequency sub-carrier is passed through a cosine or sine shaping filter. As a result, the end of filtering operation is come up with a frequency sub-carrier of Orthogonal Generalized Frequency Division Multiplexing (OGFDM). Digital Hilbert Filters (DHF) are used to multiplex / de-multiplex $K$ BW flexible frequencies of subcarriers. Throughout this paper, every pair of OGFDM filtered frequencies of subcarriers operates under the same frequency carrier but with different orthogonal phase will be called a "chunk". In synthesis level, all the generated chunks are combined digitally. All the chunk manipulations at the transmitter or receiver side will be referred to in this paper as a "Digital Chunk Processing" (DCP). The output of the DCP level is then passed through a particular Digital to Analog Converter (DAC). After converting to the analog domain, the resulted signal which is exponentially represented as $\left(e^{j 2 \pi f t}\right)$ is transmitted by the antenna. As shown in Fig. 2, at the receiver side the signal detected by the antenna is passed through an Analog to Digital Converter (ADC). In the DCP stage, decomposition, which is the inverse of the synthesis operation, is applied on the received digital signal. The processed signal is similarly fragmented to provide different chunks, where each chunk is filtered by either a cosine or sine matching filter to achieve the corresponding frequency sub-carrier, i.e., use the DHF to de-multiplex frequencies of subcarriers. Subsequently, the matched frequencies of subcarriers resealed from the DCP stage are down-sampled by a factor of $K$, denoted as $(K \downarrow)$, removing the $K-1$ zeros between adjacent samples. Thereafter, the downsampled digital subcarriers are converted back to the frequency domain. Finally, all digital subcarriers are recovered after the BPSK demodulation. The core of the OGFDM system is using the DHF to combine and isolate different digital subcarriers orthogonally. Depending on Hilbert transform properties, the shaping and matching filters can be designed and the orthogonality is achieved between them. According to the definition, The Hilbert transform $\hat{w}(t)$ of a signal $w(t)$ is defined as following [12]:

$$
\hat{w}(t)=w(t) \otimes \frac{1}{\pi t}
$$

Where $\otimes$ represents the convolution operation between $w(t)$ and the signal $\frac{1}{\pi t}$ in time domain. In Fourier transform the signal $\frac{1}{\pi t}$ is represented as $-\operatorname{sgn}(f)$ [12], where

$$
-\operatorname{sgn}(f)=\left\{\begin{array}{ll}
-j & \text { if } f>0 \\
0 & \text { if } f=0 \\
j & \text { if } f<0
\end{array}\right\}
$$

In addition, if Fourier transform of $w(t)=W(f)$, then $\hat{w}(t)$ has a Fourier transform $\hat{W}(f)$ which is equivalent to $-\operatorname{sgn}(f) W(f)$. Accordingly, the Hilbert transformation in the frequency domain is easier to implement than it is in the time domain. Using Hilbert transformation, the amplitude of the processed signal is not changed and only the phase is

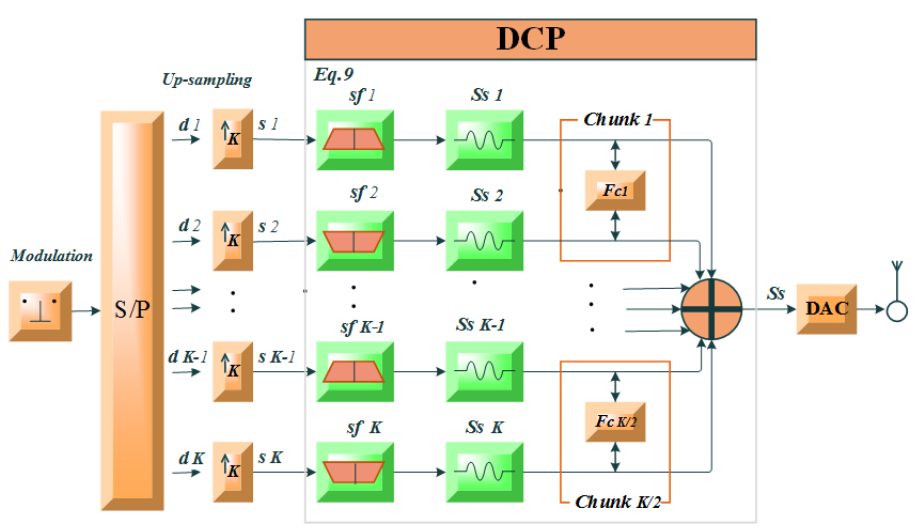

Fig. 1. The block diagram for the proposed OGFDM transmitter.

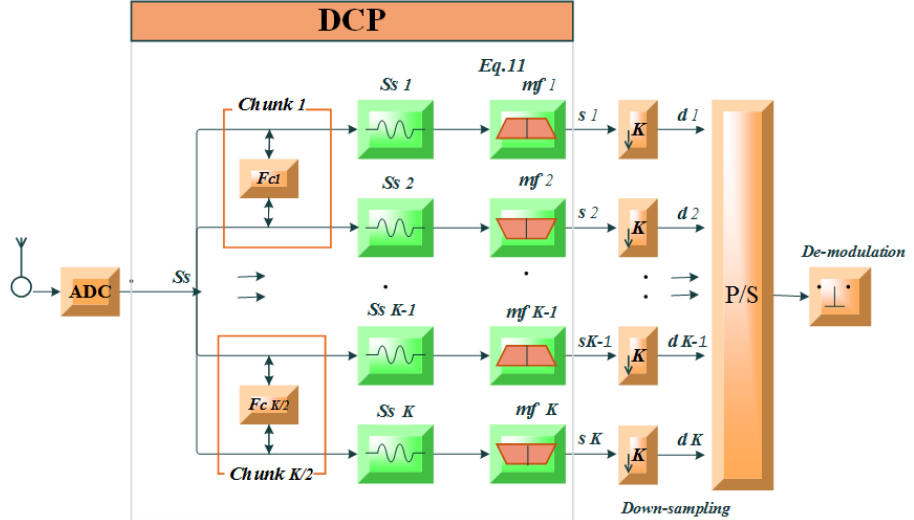

Fig. 2. The block diagram for the proposed OGFDM receiver. 
actually changed. The positive frequencies are multiplied by $-j$ while negative frequencies are multiplied by $j$ which are corresponding to phase change of $-\pi / 2$ and $\pi / 2$ respectively, where the Hilbert transform exchanges the real and imaginary parts of the signal during the phase change operation. The key property of Hilbert transform is, if $w(t)$ is a signal, then it is orthogonal to its Hilbert transform $\hat{w}(t)$ due to the zero value of the integration between them as follow [12]:

$$
\int_{-\infty}^{\infty} w(t) \hat{w}(t) d t=0
$$

According to the orthogonal facility, it can be concluded that relation between the proposed cosine and sine filters is orthogonal as $\sin (t)$ represents the Hilbert transform of $\cos (t)$. Achieve the orthogonality between adjacent filters makes them able to share the same central frequency of the filter $f c$, but double the computational complexity for it due to the dual number of multiplications for each output point [13]. To manipulate such issue, modern signal processors are fully capable to filter more than one independent input signals per clock event without the need of additional hardware resources [14]. Thus, employ the DHF with the OGFDM does not increase the overall computational complexity of filtering operation, since extra multiplication operations are accomplished simultaneously. To recover the original signal from its Hilbert transform, inverse Hilbert transform is used, thus $w(t)$ is retrieved from $\hat{w}(t)$ by applying the Hilbert transform twice and reversing the result as the following [12]:

$$
w(t)=-\hat{w}(\hat{w}(t))
$$

The main principle of the matching filters is clearly presented by equation (4). In this proposed design the main filter (cosine-filter) operates in-phase with the signal and it's the Hilbert transform or (sine-filter) is functioning in the quadrature mode. In the transmitter side, a set of parallel various shaping filters is adopted which have finite impulse responses $s f_{a}$. The transmitted signal is resulted from summating all transmitted frequencies of subcarriers in the digital domain, which is expressed as [15]:

$$
S s(t)=\sum_{a=1}^{K} s_{a}(t) \otimes s f_{a}(t)
$$

Where $S s(t)$ represents the convolution between the input sub-carrier $s_{a}(t)$ and the impulse response $s f_{a}(t)$ of the applied $a^{t h}$ filter for $K$ transmitted frequencies of subcarriers. The multiplexed OGFDM signal $S s(t)$ is passed through a single DAC to prepare it for transmission by a wireless transmission system. In the receiver side, a corresponding set of different matching filters was adopted with impulse responses $m f_{b}$. The fetched frequency sub-carrier after applying $b^{t h}$ matching filter can be expressed by [15]:

$$
s_{b}(t)=\left[\sum_{a=1}^{K} s_{a}(t) \otimes s f_{a}(t)\right] \otimes m f_{b}(t)
$$

Depending on (6), the output frequency sub-carrier $s_{b}(t)$ can be recovered if the convolution happened between the same order of shaping and matching filter, i.e., when $a=b$ the impulse response of their convolution must satisfy by [15]:

$$
s f_{a}(t) \otimes m f_{b}(t)=\delta\left(t-t_{0}\right)
$$

Where $t_{0}$ refer to the probable delay of each filtered frequency sub-carrier. Otherwise, the resulted frequency subcarrier will suffer from undesired interferences and be unrecovered, i.e., when $a \neq b$ the impulse response of their convolution will achieve as [15]:

$$
s f_{a}(t) \otimes m f_{b}(t)=0
$$

As per Fig. 1, the impulse responses of the applied Hilbert pair shaping filters satisfy the following [15]:

$$
\begin{aligned}
& s f_{1}(t)=g(t) \cos \left(2 \pi f_{c} t\right) \\
& s f_{2}(t)=g(t) \sin \left(2 \pi f_{c} t\right)
\end{aligned}
$$

Where $g(t)$ represents the baseband pulse, which has a square-root raised-cosine form expressed as [15]:

$$
g(t)=\frac{\sin [\pi(1-\alpha) \gamma]+4 \alpha \gamma \cos [\pi(1+\alpha) \gamma]}{\pi \gamma\left[1-(4 \alpha \gamma)^{2}\right]}
$$

Where $\gamma=t / \Delta t, \alpha$ represents the excess of filter BW and $\Delta t$ is the time sampling interval. It has been clear from Fig. 2 that the corresponding matching filters for Hilbert pair filters in (9), is represented by [15]:

$$
\begin{aligned}
& m f_{1}(t)=s f_{1}(-t) \\
& m f_{2}(t)=s f_{2}(-t)
\end{aligned}
$$

The impulse response of each chunk in the OGFDM system depends, in particular, on its determined filter pair shaping, matching. The range of $\alpha$ factor varies between 0 and 1 . Based on the impact of this factor, the frequency response of baseband pulse changes from a uniform to a pure squareroot raised-cosine. To satisfactorily reduce the complexity of digital filters with acceptable system BER, the number of digital filter coefficients should be perfectly estimated [15]. From Digital Signal Processing (DSP) perspective, the discrete sequences of data, which are used in the input and output of transceiver system, are resulted from sampling equivalent continuous signal at sampling interval equivalent to $\Delta t$. In OGFDM transceiver model, the period of time $\rho$ required for transmitting $K$ samples in each OGFDM symbol is equivalent to $K \Delta t / 2$. The speed of the entire transmission system can be determined by the sampling rate of the applied converter $F_{D A C / A D C}$, in addition, the sampling rate of shaping and matching filters synchronizes with the applied $F_{D A C / A D C}$. Moreover, dividing the $F_{D A C / A D C}$ by a number of applied filters determines the optimal sampling rate for each OGFDM frequency sub-carrier. According to the Shannon, the channel capacity is defined by the maximum number of transmitted bits during one iteration of time with a vanishingly small 
probability of errors [16]. In this proposed system, the channel capacity of each OGFDM signal is given by:

$$
\text { Cap }=\frac{\sum_{\iota=1}^{k} n_{\iota}}{\rho}
$$

Where $n_{\iota}$ represents the maximum number of bits buried by each frequency sub-carrier. The total channel capacity of the transmission system can be produced by accumulating sub capacities of all transmitted frequencies of subcarriers for one slide of time. In this paper, a Gaussian channel is assumed in the presence of Additive White Gaussian Noise (AWGN). The received signal $R$ is composed of the transmitted input signal $T$ multiplied by the response of the channel $E=1$ and combined with $N$ which represents here the AWGN as follow [17]:

$$
R=E T+N
$$

The power of the white noise is uniformly distributed across the frequency band of the transmission system. On the receiver side, power constraint is the main limit in communication signalling system, which is required to determine the transmission through the channel and obtain a more realistic system as the following [17]:

$$
\frac{1}{L} \sum_{i=1}^{L} x_{i}^{2} \leq P
$$

Where $P$ represents a maximum received power for $L$ transmitted number of samples. Depending on equation (14), the throughput of the transmission can be regulated by making use of the relation between the normalized power of the signal and the probable power of the AWGN. Signal to Noise Ratio (SNR) is changed according to applied modulation format. Whenever a modulation format is increased the required SNR is also increased to recover the received signal, i.e., without increasing the SNR, the distance between adjacent points on the constellation table is decreased and it is very difficult to decide a correct position for a transmitted complex number of data at the receiver side [18]. For a given transmitted power under different modulation format, there is a corresponding SNR, which in return regulates the performance in terms of the BER and channel capacity of a transmission.

\section{OGFDM TECHNIQUE IMPLEMENTATION}

In this part, the numerical simulation is demonstrated for the proposed single carrier OGFDM. It is considered for two frequencies of subcarriers utilizing the orthogonality of the DHF for a single frequency centre and with different modulation formats. The performance of transmission, in terms of channel capacity and the BER, is experimented under the condition stated in Table I. Since, the DHF is the core of the proposed transceiver system, in this experiment, the key parameters of the applied filters should be perfectly fixed according to the highest rate of channel capacity at accepted index of error. As a result, the optimal value for each parameter of the proposed digital filter has been undertaken for a maximum level of achieved channel capacity in a wireless electrical back-to-back system. To explain more about these important parameters, it is clear from Fig. 3 that the BER of transmission system can be improved by increasing the number of applied coefficients. Hence, the robust filters have the ability to adapt to different levels of noise depending their dynamic range of coefficients number. Touching with the relationship between the orthogonal filters, it is shown from Fig. 4, that the optimum level of cross-talk between the orthogonal filters for the same centre of frequency can be achieved with $\alpha=0.1$. Thus, the $\alpha$ factor indicates the optimised level of BW access for each working pair of the DHF. The evaluation of both the number of filter coefficients and $\alpha$ factor explain the crucial impact of the filter parameters on the overall system performance in terms of channel capacity and the BER. In terms of passband ripples of the utilized digital filters, it can be seen in Fig. 5, that the frequency responses of the herein proposed orthogonal filters are randomly variant showing clearly that both the cosine and sine-filters have an oscillatory construction in the frequency domain, hence, the vacillated frequency responses of these employed filters can attenuate the filtering process for the convoluted subcarrier unless managed well. To address this issue, the wobbling behaviour in frequency response can be minimized by increasing the number of filter coefficients. However, increasing the number of coefficients led to rising the computational complexity of the applied filter. For this reason, the optimal number of coefficients that achieves the accepted limits of BER should be estimated accurately. After configuring the significant parameters of the utilized filters, it is shown from Fig. 6, that the proposed single carrier OGFDM system exhibits better performance in terms of the channel capacity of transmitted chunks and BER than the standard GFDM. Thus, the new design has the ability to double the transmission rate of each chunk due to the orthogonal attributes of the employed advanced filters. Hence, each frequency carrier in the proposed design affords a dual amount of data compared with the candidate GFDM. In addition, this extra capacity resulted from employing the advanced filters for non-orthogonal frequencies of subcarriers, i.e., achieve the orthogonality without involving the Fourier Transform. Moreover, a resident $2 \mathrm{~dB}$ gain between channel capacities of the proposed OGFDM and candidate GFDM for different modulation formats is achieved. This gained increment in the

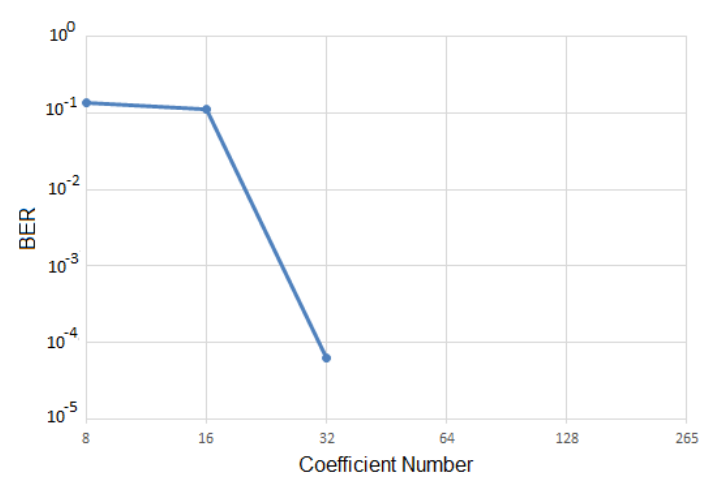

Fig. 3. Effect of filter coefficients on system performance of BER. 


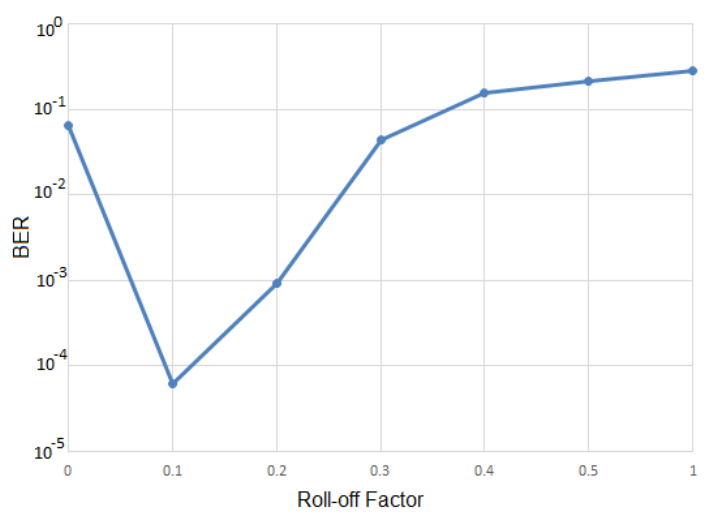

Fig. 4. Effect of $\alpha$ on system performance in terms of BER.

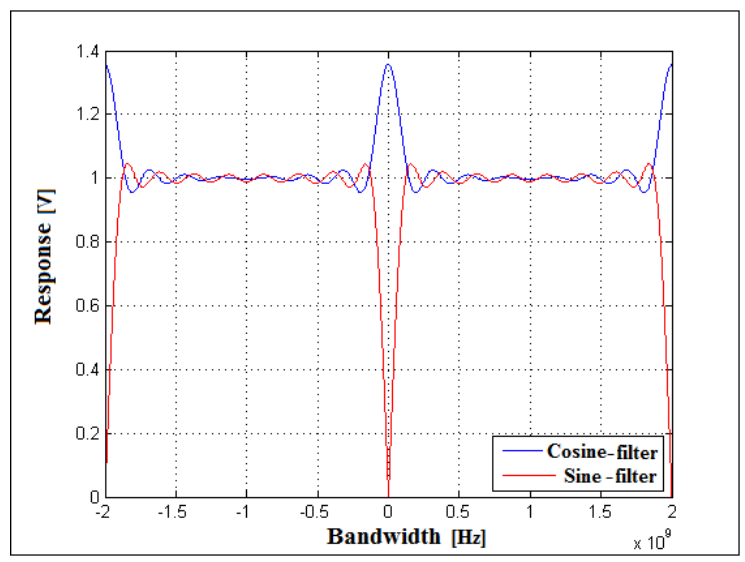

Fig. 5. Combination of cosine and sine filter responses in frequency domain.

OGFDM channel capacity comes from the difference in phase of the advanced orthogonal filters for each carrier frequency involved in the new design. Furthermore, as it is shown in Fig. 7, the calculated SNR for the proposed single carrier OGFDM with highest modulation format shows the effect of adding the AWGN on filtered frequencies of subcarriers. In addition, the similarity between the required SNR for both the OGFDM and GFDM makes the herein proposed technique capable to achieve a dual channel capacity with the same accepted

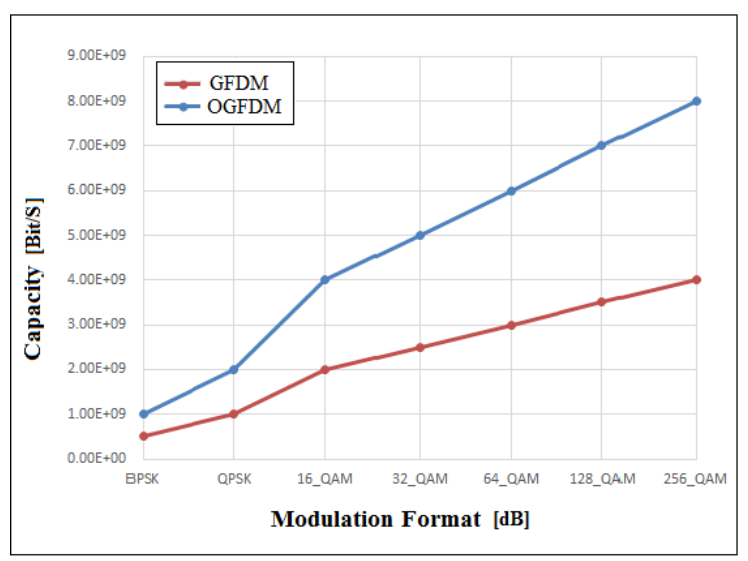

Fig. 6. Doubled channel capacity of each transmitted OGFDM chunk.

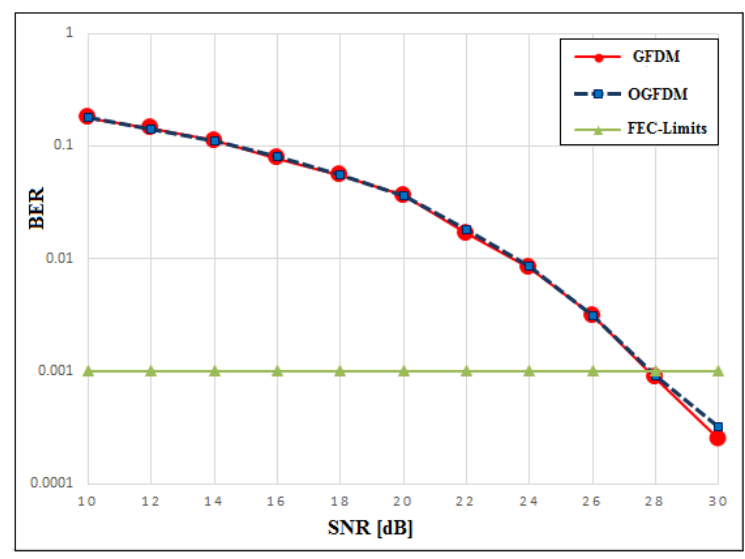

Fig. 7. Similar SNR for both OGFDM and GFDM.

TABLE I. SYSTEM PARAMETERS FOR THE OGFDM

\begin{tabular}{|c|c|}
\hline Parameter & Value \\
\hline No. of frequency centres & 1 \\
\hline$F_{D A C / A D C}$ & $4 \mathrm{GHz}$ \\
\hline Filter coefficients & 32 \\
\hline$\alpha$ & 0.1 \\
\hline System mode & Single carrier \\
\hline Sampling factor & 2 \\
\hline Modulation Format & BPSK - 256 QAM \\
\hline OGFDM symbols & 2000 \\
\hline Filter type & Hilbert filter \\
\hline
\end{tabular}

levels of the BER. Ultimately, the accomplished results of this established experiment demonstrate that the performance of proposed single carrier OGFDM with DHF in terms of the channel capacity and BER far outweigh the performance of the candidate waveform GFDM utilizing the same conditions.

\section{CONCLUSION}

In this paper, a novel OGFDM system for improving the transmission channel capacity for future Mobile networks has been proposed, investigated and evaluated in a wireless electrical back-to-back model for the first time. The developed OGFDM technique resulted from the optimal combination between the main aspects of the GFDM and OFDM in the frequency and time domain. The core of the OGFDM system is depending the advanced Hilbert filters to combine and isolate frequencies of subcarriers orthogonally. This alteration leads to double the channel capacity of each transmitted frequency carrier at accepted limits of the BER and without any extra computational complexity. Hence, promoting the filtering operation improves the performance in terms of channel capacity and the BER. The experimental work has shown 2 $\mathrm{dB}$ gain between the channel capacity of the OGFDM and the conventional GFDM comes from applying the proposed filters in the OGFDM design for different modulation formats. In addition, the similarity between the required SNR for both the OGFDM and GFDM makes the proposed technique able to achieve the double in channel capacity with the same limits of BER. Moreover, the wobbling behavior in the frequency response of the applied filters can be minimized by relying on a trade-off relation between numbers of coefficients and the complexity of the filter. Finally, its flexibility in tackling the disadvantages of the current candidate waveforms presents it as a promising solution for the future demands of wireless 
Mobile, in particular, high channel capacity applications.

\section{ACKNOWLEDGMENT}

This research was funded by the ministry of higher education and scientific research, Republic of IRAQ- Scholarship (2633).

\section{REFERENCES}

[1] R.Thandaiah, M.Benisha, V.Thulasi, "Millimeter wave for $5 G$ Mobile communication application", IEEE Int. Conf. 2016.

[2] M. Agiwal, A. Roy, N. Saxena, "Next generation $5 G$ wireless networks: A comprehensive survey ", IEEE Commun. Sur. Tut, Feb. 2016.

[3] A. Aijaz, "Towards 5G-enabled Tactile Internet: Radio resource allocation for haptic communications", IEEE Commun. Conf., Sep. 2016.

[4] X. Li, J. Rao, H. Zhang "Carrying MTC service in 5G - A network management perspective", IEEE Veh. Tech. Conf., Sept.2016.

[5] S. Stern, Robert F., H. Fischer, "OFDM vs. single-carrier modulation: A new view on the PAR behaviour", IEEE 18th Int.OFDM Workshop, Aug. 2014.

[6] N. Michailow, M. M., "Generalized frequency division multiplexing for 5th generation cellular networks, IEEE Trans. Commun., Sept. 2014.

[7] H. Kim, T. Rautio, "Weighted selective mapping algorithm for FBMCOQAM systems", IEEE Int. Conf. 2016.

[8] P. Naga, C. Santhi, "UFMC: The 5G modulation technique", IEEE Int. Conf., Dec. 2016.

[9] P. Weitkemper, J. Bazzi, K. Kusume, "On regular resource grid for filtered OFDM", IEEE Commun. Let., Dec. 2016.

[10] S. Han, Y. Sung, Y. Lee "Filter design for generalized frequencydivision multiplexing", IEEE Trans. Sig. Pro., April 2017.

[11] J. Demel, C. Bockelmann, A. Dekorsy, "Evaluation of a software defined GFDM implementation for industry 4.0 applications", IEEE Int. Conf., 2017.

[12] Alexander D. Poularikas, "The transforms and applications handbook", USA: CRC Press LLC. 2000.

[13] V. K. Ingle, J. G. Proakis , "Digital signal processing using MATLAB", 3rd Ed. USA, 2012.

[14] Z. Jiang, S. Mao, "Energy Delay Tradeoff in Cloud Offloading for Multi-Core Mobile Devices", USA, IEEE Access, 2015.

[15] L. Tao, Y. Wang, Y. Gao , "Experimental demonstration of $10 \mathrm{~Gb} / \mathrm{s}$ multilevel carrier-less amplitude and phase modulation for short range optical communication systems", Opt. Exp.2013.

[16] G. Im, D. Harman, G. Huang , "51.84 Mb/s 16-CAP ATM LAN Standard", IEEE J.Selected Areas Commun. , 1995.

[17] M.Ghogho, D. McLernon, "Channel estimation and symbol detection for block transmission using data- dependent superimposed training", IEEE Sig. Proc., March 2005.

[18] Y. Liang, K. Chen, G. Li, and P. Mahonen, "Cognitive radio networking and communications", IEEE Trans. Veh. Technol., Sep. 2011. 